\title{
A NOTE ON MINIMAL COVERS FOR SOFIC SYSTEMS ${ }^{1}$
}

\author{
MIKE BOYLE, BRUCE KITCHENS AND BRIAN MARCUS
}

\begin{abstract}
We characterize the sofic systems which have minimal subshift-of-finitetype covers and derive some consequences
\end{abstract}

0. Introduction. For relevant background, see $§ 1$. By a factor map, we mean a continuous onto shift commuting map between two symbolic systems. By definition, a sofic system $S$ is a symbolic system which is a factor of a shift of finite type (SFT). In the language of automata theory, sofic systems are regular languages. A minimal cover for $S$ is a SFT, $\Sigma_{A}$, and a factor map $\pi: \Sigma_{A} \rightarrow S$ such that any other factor map $\varphi: \Sigma_{B} \rightarrow S$ (from a SFT onto $S$ ) must factor through $\pi$ :

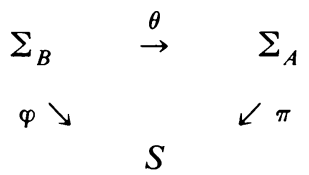

Our main result (Corollary 13) is that a sofic system $S$ has a minimal cover if and only if $S$ is almost of finite type (i.e., $S$ can be realized by a factor map which makes identifications on only a proper closed subset of an SFT). This concept (AFT) was introduced in $[\mathbf{M}]$ for coding purposes; the theory of modulation codes provides many natural AFT examples. The results herein give an effective method (Corollary 11) of determining whether or not a given sofic system is AFT; this same criterion was found independently by M. Nasu [N2] and W. Krieger (private communication).

Before proceeding to the main result, we review the "future cover" [F1] and note that this cover is minimal (for all sofic systems) among a special class of maps: right closing maps (this follows readily from Fischer's result [F1]). From this minimality result, we recover part of W. Krieger's theorem (Theorem 6) that the "future cover" is a topological conjugacy invariant of the underlying sofic system.

While some of the results of this paper have been discovered independently, one purpose of this paper is to show how they can be obtained from the perspective of minimal covers.

1. Background. We first briefly summarize the relevant background. See [AM, §3] for more details.

Let $\mathscr{A}$ denote $\{1, \ldots, n\}$ with the discrete topology. The full $n$-shift is the space $\mathscr{A}^{Z}$ with the product topology. So, a point in the full $n$-shift is simply a bi-infinite

Received by the editors October 3, 1984. This paper was presented at the Annual AMS Meeting. January 1985, Anaheim, California.

1980 Mathematics Subject Classification. Primary 28D20; Secondary 54H20, 58F20.

${ }^{1}$ Partially supported by NSF Research Grant No. MCS- 8301246. 
sequence of integers in $\mathscr{A}$. One defines the shift map

$$
\sigma: \Sigma_{n} \rightarrow \Sigma_{n},
$$

$\boldsymbol{\sigma}(x)=y$ where each $y_{i}=x_{i+1}$. One easily sees that $\sigma$ is continuous.

By a subshift (or symbolic system), we mean the restriction of $\sigma$ to a closed $\sigma$-invariant subset $\Lambda$ of $\Sigma_{n}$. This means that there is a collection (possibly infinite) $C$ of finite words in the alphabet $\mathscr{A}$ such that ( $x$ belongs to $\Lambda$ ) $\Leftrightarrow$ (each finite block of $x$ belongs to $C$ ).

Note that since a subshift $\Lambda$ is defined to be shift invariant, the shift map $\sigma$ naturally restricts to a map $\sigma: \Lambda \rightarrow \Lambda$. Sometimes, we use $\Lambda$ to refer to either $\Lambda$ or $\Lambda$ together with $\sigma / \Lambda$.

Let $\Lambda_{1}$ and $\Lambda_{2}$ be two subshifts with possibly different alphabets. A factor map from $\Lambda_{1}$ to $\Lambda_{2}$ is a continuous onto map $\pi: \Lambda_{1} \rightarrow \Lambda_{2}$ such that $\pi \sigma=\sigma \pi$.

While the definition of a factor map has an abstract form, it is really a very concrete idea: it is simply a sliding block code. In general, a $k$-block factor map $\pi$ is a sliding block code generated by a map

$$
\begin{gathered}
\pi^{*}:\left\{k \text {-blocks of } \Lambda_{1}\right\} \rightarrow\left\{\text { symbols of } \Lambda_{2}\right\}, \\
\pi\left(\left(x_{i}\right)\right)=\cdots \pi^{*}\left(x_{1} \cdots x_{k}\right) \pi^{*}\left(x_{2} \cdots x_{k+1}\right) \cdots .
\end{gathered}
$$

Factor maps which are 1-1 and onto are called (topological) conjugacies. They play a very special role. If a conjugacy from $\Lambda_{1}$ to $\Lambda_{2}$ exists, we say that $\Lambda_{1}$ and $\Lambda_{2}$ are conjugate. The idea is that two conjugate subshifts are essentially the same even if they produce literally different sequences.

Let $\Lambda$ be a subshift and let $k$ be a positive integer. Let $C_{k}$ denote the set of all $k$-blocks of $\Lambda$. Define

$$
\begin{gathered}
\phi: \Lambda \rightarrow\left(C_{k}\right)^{z}, \\
\phi\left(\cdots x_{-1} x_{0} x_{1} \cdots\right)=\cdots\left(x_{-1} \cdots x_{k-2}\right)\left(x_{0} \cdots x_{k-1}\right)\left(x_{1} \cdots x_{k}\right) \cdots .
\end{gathered}
$$

Note that the blocks here overlap. The image of $\phi$ is a subshift, conjugate (via $\phi$ ) to $\Lambda$. This subshift is called the higher $k$-block system for $\Lambda$ and is one of many different and convenient ways that we can recode a subshift.

We are interested in subshifts of finite type and sofic systems. A subshift $\Lambda$ is of finite type (SFT) if there is a positive integer $k$ and a collection of $k$-blocks $C$ such that

$$
\Lambda=\left\{x \in \mathscr{A}^{z}: \forall i x_{i+1} x_{i+2} \cdots x_{i+k} \in C\right\} .
$$

In other words, $\Lambda$ is the set of points all of whose $k$-blocks are prescribed by $C$.

If $k=2$, then one constructs an $n \times n$ matrix

$$
A_{i j}=\left\{\begin{array}{l}
1 \text { if } i j \in C \\
0 \text { if } i j \notin C
\end{array}\right\} .
$$

In this case $(k=2)$, the SFT is denoted $\Sigma_{A}$. By a simple recoding (via the higher $k$-block system), every SFT may be described as a $\Sigma_{A}$ (with perhaps a much larger set of states). If $i j \in C$, we say that there is an (allowable) transition $i \rightarrow j$. 
As is standard, one may represent a SFT $\Sigma_{A}$ as the set of all bi-infinite walks on a directed graph as follows: The states are the elements of $\mathscr{A}$; one draws an edge from $i$ to $j$ if and only if $A_{i j}=1$. Thus, the point $x=\left(\cdots x_{-1} x_{0} x_{1} x_{2} \cdots\right)$ corresponds to a walk which at time $i$ is at state $x_{i}$. For example, if $A=\left(\begin{array}{ll}1 & 1 \\ 1 & 0\end{array}\right)$ then the graph is

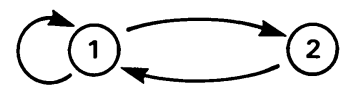

One says that a subshift $\Lambda$ is sofic if there is a SFT $\Sigma_{A}$ and a factor map $\pi$ from $\Sigma_{A}$ onto $\Lambda$. Of course, every SFT is sofic (let $\pi=$ identity), but sofic systems are much more general.

In the definition of sofic system, by replacing $\Sigma_{A}$ by a higher block system, one may assume that $\pi$ is a 1-block map, or equally well, a 2-block map. From this point of view then, a sofic system is a subshift obtained by labelling the vertices if 1-block (or edges if 2-block) of a directed graph. For example, the edge labelling

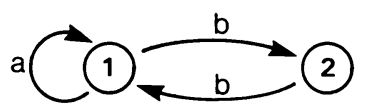

generates a sofic system called the even system ( $b$ 's appear in blocks of even length).

The entropy $h(\Lambda)$ of a subshift $\Lambda$ is simply the asymptotic growth rate of the number of $k$-blocks of $\Lambda$ (as $k \rightarrow \infty)$. For an irreducible SFT $\Sigma_{A}, h\left(\Sigma_{A}\right)=\log (\lambda)$ where $\lambda$ is the largest eigenvalue of $A$.

An SFT $\Sigma_{A}$ is called irreducible if its graph is strongly connected. In the case that $\Sigma_{A}$ is not irreducible, it may be studied in terms of its "irreducible components."

2. Right resolving covers. We assume that all of our sofic systems are transitive with periodic points dense; every such sofic system is a factor of an irreducible SFT (see [CP2]).

Definition. Let $\pi_{1}: S_{1} \rightarrow S_{1}^{\prime}$ and $\pi_{2}: S_{2} \rightarrow S_{2}^{\prime}$ be onto factor maps between sofic systems. We say $\pi_{1}$ is topologically conjugate to $\pi_{2}$ if there exist topological conjugacies $\varphi$ and $\psi$ (of the underlying shifts) such that

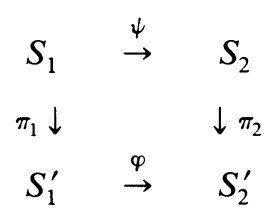

commutes.

Definitions [AM,K]. A factor map $\pi: \Sigma_{A} \rightarrow S$ from a SFT onto a sofic system is right closing if whenever $x$ and $y$ are negatively asymptotic points in $\Sigma_{A}$ and $\pi(x)=\pi(y)$, then $x=y$. A 1-block factor map $\pi$ is called right resolving if whenever $s_{1} s_{2}$ is a 2-block in $S$ and $i$ is a symbol in $\Sigma_{A}$ with $\pi(i)=s_{1}$, then there is at most one symbol $j$ in $\Sigma_{A}$ such that $i j$ is an allowable 2-block in $\Sigma_{A}$ and $\pi(i j)=s_{1} s_{2}$. 
Note. If $S$ is a SFT, then in the above definition "at most one" can be replaced by "exactly one."

Clearly any right resolving map is right closing, but up to a recoding, the converse is true as well. A precise statement of this is as follows:

Proposition 1 (ESSENTIALlY IN [K]). Every right closing factor map $\pi: \Sigma_{A} \rightarrow S$ is topologically conjugate to a right resolving factor map $\pi^{\prime}: \Sigma_{A^{\prime}} \rightarrow S$.

Proof. By recoding, we may assume that $\pi$ is a 1-block map. Since $\pi$ is right closing, a standard compactness argument shows that there is a largest integer $M$ for which there exists a pair of $M$-blocks, $x_{1} \cdots x_{M}$ and $y_{1} \cdots y_{M}$, such that $x_{1}=y_{1}$, $x_{2} \neq y_{2}$ and $\pi\left(x_{1} \cdots x_{M}\right)=\pi\left(y_{1} \cdots y_{M}\right)$. We then define $\Sigma_{A^{\prime}}$ by the partition of $M$-blocks on $\Sigma_{A^{\prime}}$ :

$$
x_{1} \cdots x_{M} \sim y_{1} \cdots y_{M} \Leftrightarrow \pi\left(x_{1} \cdots x_{M}\right)=\pi\left(y_{1} \cdots y_{M}\right) \quad \text { and } x_{1}=y_{1} .
$$

We define $\pi^{\prime}: \Sigma_{A^{\prime}} \rightarrow S$ by $\pi^{\prime}\left(\left[x_{1} \cdots x_{M}\right]\right)=\pi\left(x_{M}\right)$ where [ ] denotes the equivalence class. The maximality condition on $M$ forces $\pi^{\prime}$ to be right resolving.

REMARK. One can define the notions of right closing and right resolving for factor maps between arbitrary subshifts, and with a little more work, one can get the result above in this generality.

Similarly, one has the notions of left closing and left resolving.

Proposition 2 [CK]. Every sofic system $S$ can be realized as the image of a SFT by a 1-1 a.e. right resolving factor map. (Similarly, there is a 1-1 a.e. left resolving factor map which realizes $S$.)

We review the construction of Proposition 2 as follows: Let $S$ be a sofic system and let $u$ be a block in $S$. The follower set is defined:

$$
\mathscr{F}(u)=\{\text { rays } w \text { in } S: u w \text { is in } S\} .
$$

Let $\Sigma_{s}^{f}$ be the SFT whose alphabet is

$$
\{(i, \mathscr{F}(u)): i \text { is a symbol in } S \text { and } u \text { is a block in } S\}
$$

and transitions are

$$
(i, \mathscr{F}(u)) \rightarrow(j, \mathscr{F}(v)) \quad \text { iff } \quad \mathscr{F}(v)=\mathscr{F}(u j) .
$$

The map $\pi_{s}^{f}: \Sigma_{s}^{f} \rightarrow S$ defined by $\pi_{s}^{f}((i, \mathscr{F}(u))) \equiv i$ is the 1-1 a.e. right resolving factor map and the pair $\left(\Sigma_{s}^{f}, \pi_{s}^{f}\right)$ is called the "future cover."

Definition. Let $S$ be a sofic system. A magic word is a block $w$ in $S$ such that whenever $u$ and $v$ are blocks in $S$ and $u w$ and $w v$ are in $S$, then so is $u w v$. We leave the following as an exercise (see also [Kr, Lemma 2.7]):

Proposition 3. $\Sigma_{s}^{f}$ has a unique irreducible component of maximal entropy-namely, the component defined by the symbols

$$
\left\{(j, \mathscr{F}(w)): w=w_{1} \cdots w_{n} \text { is a magic word and } w_{n}=j\right\} .
$$

We Let $\Sigma_{s}^{+}$denote this component and $\pi_{s}^{+}: \Sigma_{s}^{+} \rightarrow S$ the restriction of the future cover to $\Sigma_{s}^{+}$. This map is onto. 
Proposition 4. Let $\varphi: \Sigma_{B} \rightarrow S$ be a right closing factor map. Then $\varphi$ factors through $\pi_{s}^{+}$, i.e., there exists $\theta$ such that

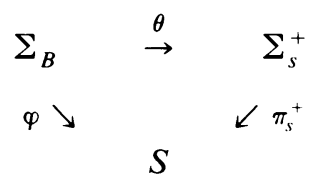

commutes.

Proof. By Proposition 1, we may assume that $\varphi$ is right resolving. Now, in the terminology of [F1], $\varphi$ represents a "Shannon Graph." In [F1], it is then shown that any "Shannon Graph" can be reduced to a "minimal Shannon Graph" (see the proofs of Theorem 4(iii) and Lemma 3 of [F1]). There, minimal means minimal in terms of the number of vertices, but one easily checks that one has minimality in the sense of the commutative diagram above. The rough idea is that for each symbol $i$ in $\Sigma_{B}$, there is a block $w$ in $S$ such that $\varphi(\mathscr{F}(i))=\mathscr{F}(w)$. One then defines $\theta$ by $\theta(i)=(\varphi(i), \varphi(\mathscr{F}(i)))$ and checks that this is well defined.

Proposition 5. Suppose $\pi_{s}^{+}=\pi_{2} \cdot \pi_{1}$ where the Range of $\pi_{1}=$ Domain of $\pi_{2}$ is a SFT. Then $\pi_{1}$ is 1-1 everywhere.

Proof. Let $\Sigma_{B}$ denote the range of $\pi_{1}$. We have by hypothesis

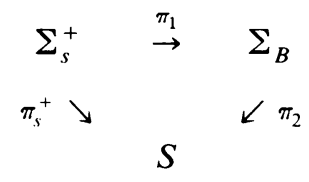

By Proposition 4, we have a diagram

$\begin{array}{lll}\Sigma_{B} & \stackrel{\theta}{\rightarrow} & \Sigma_{s}^{+} \\ \pi_{2} \searrow & & \swarrow \pi_{s}^{+} \\ & S & \end{array}$

Putting these together, we get a diagram

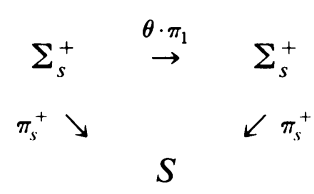

But since $\pi_{s}^{+}$is $1-1$ a.e., $\theta \cdot \pi_{1}=$ identity a.e. Since $\theta \cdot \pi_{1}$ is continuous, $\theta \cdot \pi_{1}=$ identity. So, $\pi_{1}$ is $1-1$. We now recover

THEOREM 6 (KRIEGER [Kr]). Let $S_{1}$ and $S_{2}$ be topologically conjugate sofic systems. Then $\pi_{s_{1}}^{+}$and $\pi_{s_{2}}^{+}$are topologically conjugate. 
Proof. Let $\varphi: S_{1} \rightarrow S_{2}$ be the conjugacy. There exists a map $\theta: \Sigma_{s_{1}}^{+} \rightarrow \Sigma_{s_{2}}^{+}$by Proposition 4 such that

$$
\begin{array}{ccc}
\Sigma_{s_{1}}^{+} & \stackrel{\theta}{\rightarrow} & \Sigma_{s_{2}}^{+} \\
\pi_{s_{1}}^{+} \downarrow & & \downarrow \pi_{s_{2}}^{+} \\
S_{1} & \stackrel{\varphi}{\rightarrow} & S_{2}
\end{array}
$$

Since $\pi_{s_{1}}^{+}=\left(\varphi^{-1} \cdot \pi_{s_{2}}^{+}\right) \cdot \theta$, it follows by Proposition 5 that $\theta$ is $1-1$. Thus, the diagram above is a conjugacy.

Krieger $[\mathbf{K r}]$ actually showed that the entire future cover is a conjugacy invariant.

\section{AFT systems.}

Definition. A sofic system is almost of finite type (AFT) if it is the image of an irreducible SFT via a factor map which is 1-1 on a nontrivial open set. (Equivalently, the sofic system is realized from the SFT by making identifications on only a proper closed subset.)

The AFT concept was introduced in [M] for coding purposes. The following gives an equivalent definition which is easier to work with.

Proposition 7 [M]. Let $\Sigma_{A}$ be an irreducible SFT and $\pi: \Sigma_{A} \rightarrow S$ a factor map onto a sofic system $S$. Then $\pi$ is 1-1 on an open set if and only if $\pi$ is right closing, left closing and 1-1 a.e.

Proposition 8 ([N1, Theorem 6.3]; See Also [H, Theorem 16.11]). Let $\Sigma_{A}$ and $\Sigma_{B}$ be irreducible SFT's. Let $\pi: \Sigma_{A} \rightarrow \Sigma_{B}$ be right and left closing, then $\pi$ is constant-toone.

Definition. Let $\pi: \Sigma_{A} \rightarrow S$ and $\varphi: \Sigma_{B} \rightarrow S$ be two factor maps from irreducible SFT's $\Sigma_{A}$ and $\Sigma_{B}$ onto a sofic system $S$. The fibered product of $\varphi$ and $\pi$ is the SFT

$$
\Sigma_{T}=\left\{(x, y) \in \Sigma_{A} \times \Sigma_{B}: \pi(x)=\varphi(y)\right\}
$$

together with the projection maps $\theta_{1}: \Sigma_{T} \rightarrow \Sigma_{A}, \theta_{2}: \Sigma_{T} \rightarrow \Sigma_{B}$. It is easily verified that $\Sigma_{T}$ is indeed a SFT.

The following shows that any AFT sofic system has a minimal cover:

THEOREM 9. Let $S$ be an AFT sofic system, presented by the factor map $\pi: \Sigma_{A} \rightarrow S$ where $\pi$ is 1-1 on an open set. Let $\varphi: \Sigma_{B} \rightarrow S$ be any factor map. Assume that $\Sigma_{A}$ and $\Sigma_{B}$ are both irreducible. Then $\varphi$ factors through $\pi$.

Proof. Let $\Sigma_{T}$ be the fibered product for $\pi$ and $\varphi$ :

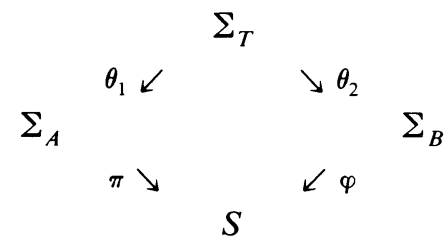


We will first show that $\theta_{2}$ is $1-1$ on some irreducible component of $\Sigma_{T}$. To see this, first observe that since $\pi$ is $1-1$ on an open set, it is right closing and left closing (Proposition 7). Thus, so is $\theta_{2}$. So, $\theta_{2}$ is finite-to-one (since, by Proposition 1 , right closing maps are conjugate to right resolving maps); thus, any irreducible component $\Sigma_{T^{\prime}}$ (of $\Sigma_{T}$ ) with maximal entropy (in $\Sigma_{T}$ ) maps (via $\left.\theta_{2}\right)$ onto a subshift of $\Sigma_{B}$ with maximal entropy in $\Sigma_{B}$. By [CP1, Theorem 3.3] this means that $\left.\theta_{2}\right|_{\Sigma_{T}}$ is onto. Now since $\theta_{2}$ is right and left closing, it follows that $\left.\theta_{2}\right|_{\Sigma_{T}}$ is constant-to-one (by Proposition 8). But what constant $K$, can this be? If $K>1$, then for each point $y \in \Sigma_{B}$, there exist two points $x, x^{\prime} \in \Sigma_{A}$ such that $(x, y)$ and $\left(x^{\prime}, y\right)$ belong to $\Sigma_{T^{\prime}}$ and hence $\pi(x)=\pi\left(x^{\prime}\right)=\varphi(y)$. Since $y$ is arbitrary and $\varphi$ is onto, this then implies that $\pi$ is at least two-to- 1 everywhere. This is contrary to the assumption that $\pi$ is $1-1$ on an open set. Thus, $K=1$, i.e., $\theta_{2}$ is invertible. Let $\theta=\theta_{1} \circ \theta_{2}^{-1}$. Then $\varphi=\pi \circ \theta$. So, if we can show that $\theta$ is a factor map, we will have shown that $\varphi$ factors through $\pi$. Well, clearly $\theta$ is continuous and shift-commuting. To see that $\theta$ is onto, first observe that since $\varphi$ is onto, $\pi$ maps $\theta\left(\Sigma_{B}\right)$ onto $S$; since $\pi$ is finite-to-one, this implies that $\theta\left(\Sigma_{B}\right)$ has maximal entropy in $\Sigma_{A}$. So by [CP1, Theorem 3.3], $\theta\left(\Sigma_{B}\right)=$ $\Sigma_{A}$.

We now derive some consequences: The following shows that an AFT sofic system can be presented as an AFT system in only one way!

Corollary 10. Let $S$ be an AFT sofic system, presented by $\pi: \Sigma_{A} \rightarrow S$ (where $\pi$ is 1-1 on an open set). Then, $\pi$ is topologically conjugate to $\pi_{s}^{+}$.

Proof. By the previous theorem, $\pi_{s}^{+}$must factor through $\pi$ :

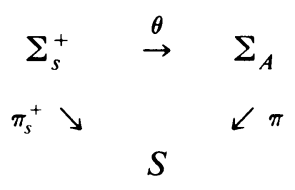

But then by Proposition 5, $\theta$ must be a conjugacy.

The following is a criterion for AFT. (It was also discovered independently by Nasu [N2, Theorem 6.1] and W. Krieger.)

Corollary 11. Let $S$ be a sofic system. Then $S$ is AFT if and only if $\pi_{s}^{+}$is left closing.

Proof. If $S$ is AFT, then by Corollary 10 and Proposition $7, \pi_{s}^{+}$is right and left closing.

Conversely, if $\pi_{s}^{+}$is left closing, then since, by construction, it is right closing and 1-1 a.e., $S$ is AFT (by Proposition 7).

LEMMA 12. Let $\Sigma_{A}$ be an irreducible SFT and let $\pi: \Sigma_{A} \rightarrow S$ be a right closing factor map. Suppose $\pi=\pi_{2} \cdot \pi_{1}$ where the (Range of $\left.\pi_{1}\right)=\left(\right.$ Domain of $\left.\pi_{2}\right)$ is a SFT. Then both $\pi_{1}$ and $\pi_{2}$ are right closing (similarly, for left closing).

Proof. Clearly $\pi_{1}$ is right closing. Then by recoding (Proposition 1), we may assume that $\pi_{1}$ is a right resolving map from $\Sigma_{A}$ onto the (Range of $\pi_{1}$ ), which is a SFT. Thus, any pair $\{x, y\}$ of negatively asymptotic points in the range of $\pi_{1}$ lifts 
(via $\pi_{1}$ ) to a pair of negatively asymptotic points $\left\{x^{\prime}, y^{\prime}\right\}$ in $\Sigma_{A}$. Now, suppose $\pi_{2}(x)=\pi_{2}(y)$; we wish to show that $x=y$ (so $\pi_{2}$ will then be right closing). Well,

$$
\pi\left(x^{\prime}\right)=\pi_{2} \cdot \pi_{1}\left(x^{\prime}\right)=\pi_{2}(x)=\pi_{2}(y)=\pi_{2} \cdot \pi_{1}\left(y^{\prime}\right)=\pi\left(y^{\prime}\right) .
$$

Since $\pi$ is right closing, $x^{\prime}=y^{\prime}$; thus, $x=y$, as desired.

REMARK. The lemma above is false without the SFT assumption on the Range of $\pi_{1}$. However, the SFT assumption on the domain of $\pi$ can be relaxed to the assumption that the domain is a sofic system, transitive with periodic points dense.

COROLlary 13. A sofic system $S$ is AFT if and only if it has a minimal cover.

Proof. Only if is Theorem 9. If $S$ has a minimal cover $\pi$, then both right and left closing covers of Proposition 2 must factor through $\pi$. Hence, by Lemma 12, $\pi$ must be both right and left closing. It must also be 1-1 a.e. since the right and left closing covers are. Thus, by Proposition 7, $S$ is AFT.

EXAMPLE. We now give a simple example of a sofic system which has no minimal SFT cover-equivalently (by Corollary 13), is not AFT. Let $S$ be the sofic system on symbols $1,2,3,4,5$ obtained by identifying $1^{a}$ and $1^{b}$ in the graph below:

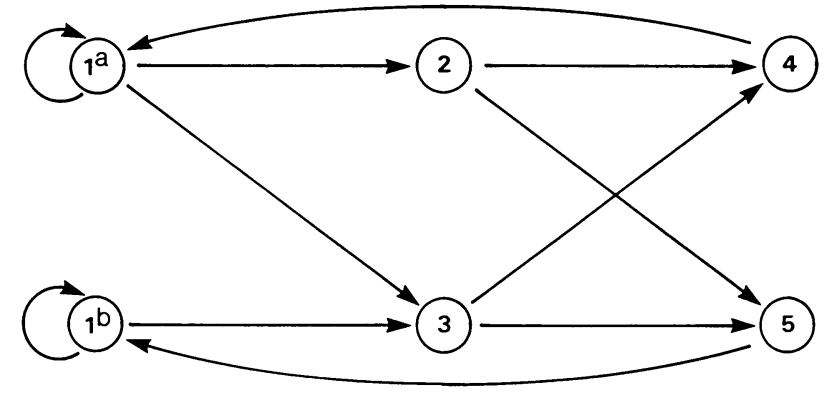

The reader can easily check that this represents the "future cover" $\pi_{s}^{+}$and the covering map is not left closing. So, by Corollary 11, $S$ is not AFT.

Actually, this example was the motivation for this paper; several years ago, one of the authors (B. Kitchens) gave a direct proof that the sofic system $S$ above has no minimal SFT cover. Later one of the other authors (M. Boyle) showed directly (in his Ph.D. thesis), that $S$ is not AFT. Now, we know that these two results are equivalent.

We finally raise the following:

QUESTION. $^{2}$ For every sofic system $S$, does there exist a finite collection of SFT 1-1 a.e. covers $\pi_{1}, \ldots, \pi_{n}$ such that every 1-1 a.e. factor map from a SFT onto $S$ factors through some $\pi_{i}$ ? Of course, if $S$ is AFT, then one needs only one $\pi_{i}$.

\section{REFERENCES}

[AM] R. Adler and B. Marcus, Topological entropy and equivalence of dynamical systems, Mem. Amer. Math. Soc., No. 219 (1979).

[CK] I. Csizar and J. Komlos, On the equivalence of two models of finite-state noiseless channels from the point of view of the output, Information Theory, Vol. 1, Proc. Colloq. Math. Soc. Janos Bolyai, 1968.

\footnotetext{
${ }^{2} \mathrm{~S}$. Williams has answered this question in the negative.
} 
[CP1] E. Coven and M. Paul, Endomorphisms of irreducible subshifts of finite type, Math. Systems Theory 8 (1974), 167-175.

[CP2] . Sofic sy'stems, Israel J. Math. 20 (1975), 165-177.

[F1] R. Fischer, Graphs and symbolic dynamics, Information Theory, Vol. 16, Proc. Colloq. Math. Soc. Janos Bolyai, 1975.

[F2] __ Sofic systems and graphs, Monatsh. Math. 80 (1975), 179-186.

[H] G. A. Hedlund, Endomorphisms and automorphisms of the shift dynamical system, Math. Systems Theory 3 (1969), 320-375.

[K] B. Kitchens, Ph.D. Thesis, University of North Carolina, Chapel Hill, N.C., 1981.

[Kr] W. Krieger, On sofic systems. I, Israel J. Math. 48 (1984), 305-330.

[M] B. Marcus, Sofic systems and encoding data, IEEE - Information Theory 31 (1985), 366-377.

[N1] M. Nasu, Constant-to-one and onto global maps of homomorphisms between strongly connected graphs, Ergodic Theory Dynamical Systems 3 (1983), 387-414.

[N2] An invariant for bounded-to-one factor maps between sofic subshifts, Ergodic Theory Dynamical Systems 5 (1985), 89-107.

[W] B. Weiss, Subshifts of finite type and sofic systems, Monatsh. Math. 77 (1973), 462-474.

Mathematical Sciences Research Institute, Berkeley, California 94720

Department of Mathematics, University of Maryland, College Park, Maryland (Current address of Mike Boyle)

IBM Research Laboratory, Yorktown Heights, New York 10598 (Current address of Bruce Kitchens)

IBM RESEARCH Laboratory, SAN JoSE, CALIFornia 95193 (Current address of Brian Marcus) 